\title{
SISTEMA REPRODUCTIVO EN CRINODENDRON PATAGUA MOL. (ELAEOCARPACEAE), UN ARBOL ENDEMICO DE CHILE CENTRAL
}

\author{
BREEDING SYSTEM IN CRINODENDRON PATAGUA MOL. \\ (ELAEOCARPACEAE), AN ENDEMIC TREE OF CENTRAL CHILE
}

\author{
Ana María Humaña \& Carlos E. Valdivia ${ }^{1}$ \\ Departamento de Ciencias Ecológicas, Facultad de Ciencias, Universidad de Chile. Casilla 653. Santiago, Chile. \\ ${ }^{1}$ Correo electrónico: cvaldiviap@yahoo.com
}

\begin{abstract}
RESUMEN
Se determinó el sistema reproductivo del árbol endémico de Chile central Crinodendron patagua, realizándose cuatro tratamientos de polinización dirigida más un control por polinización natural. Las flores de la prueba de agamospermia no fructificaron exitosamente, demostrando que la especie no es apomíctica. La prueba de autopolinización automática alcanzó un 6,9\% de fructificación, a pesar del 27,7\% de fructificación alcanzado en la prueba de autopolinización manual. La prueba de polinización cruzada alcanzó un $72,9 \%$ de fructificación exitosa, igualando el valor de fructificación en la prueba de polinización natural. De acuerdo a estos datos, C. patagua debe ser considerada como una planta autoincompatible, con un índice de incompatibilidad de 0,12, además de xenógama. De hecho, C. patagua presenta una floración masiva en primavera, dando numerosas flores blancas con forma de campana que son visitadas por himenópteros y dípteros que pueden actuar como vectores polínicos.

Palabras ClaVes: Autoincompatibilidad, polinización, semillas, xenogamia.
\end{abstract}

\section{ABSTRACT}

The breeding system of Crinodendron patagua was determined through four directed pollination trials and a control for natural pollination. An agamospermy test did not result in fruit set, indicating that this species is non-apomictic. In an automatic self-pollination test successful fruit set was $6.9 \%$ of compared with $27.7 \%$ attained in a hand self-pollination test. A cross-pollination test produced $72.9 \%$ fruiting, which did not differ from natural pollination control. Hence, $C$. patagua must be considered a self-incompatible xenogamous plant, with a self-incompatibility index of 0.12. C. patagua flowers massively in spring, with numerous white flowers visited by Hymenoptera and Diptera, which may carry pollen between flowers.

KEYWORDS: Pollination, self-incompatibility, seed set, xenogamy.

\section{INTRODUCCION}

El sistema reproductivo de las plantas contribuye a determinar los patrones de transmisión de genes y con ello la organización de la variación genética, afectando, por consiguiente, la adecuación biológica individual y poblacional (Frankel \& Galun 1977, Richards 1986, Barrett 1988). El sistema reproductivo determina las vías por las cuales se produci- rán las semillas, siendo éstas: agamospermia, autogamia, geitonogamia y xenogamia (Richards 1986).

Tanto la agamospermia como la autopolinización por autogamia y geitonogamia aumentan los niveles de homocigosis con la consecuente pérdida de adecuación biológica, aumentando también las diferenciaciones genéticas entre las poblaciones (Frankel \& Galun 1977, Charlesworth \& Charlesworth 
1987). Por su parte, la xenogamia, es decir, la polinización cruzada entre individuos distintos, promueve el flujo génico reduciendo las diferenciaciones microgeográficas incrementando las variaciones genéticas con el consecuente mayor potencial de adaptación ante eventuales cambios ambientales (Eckert \& Barrett 1994).

En plantas hermafroditas, el principal y más efectivo mecanismo para prevenir la autofertilización por autopolinización corresponde a la autoincompatibilidad genética (Barrett 1988). La autoincompatibilidad es el resultado de la expresión de un conjunto de restricciones fisiológicas que impiden a las plantas producir semillas mediante la fertilización de los óvulos con el polen producido por el mismo individuo (Barrett 1988, Richards 1986). En general, las plantas leñosas tienden a ser autoincompatibles mientras que las herbáceas, autocompatibles (Arroyo \& Squeo 1990, Barrett 1998). Esto se debería, principalmente, a que las perennes pierden adecuación ya sea en términos de reproducción o sobrevivencia, cuando la producción de semillas se ve aumentada por la autopolinización (Morgan et al. 1997).

El género Crinodendron está representado por cuatro especies leñosas distribuidas en el cono sur de Sudamérica (Bricker 1991). De éstas, dos son endémicas al territorio continental chileno: $C$. hookerianum y C. patagua (Rodríguez et al. 1983). En Chile, parte de la biología reproductiva del género ha sido abordada por Humaña \& Arroyo (datos no publicados), quienes determinaron autoincompatibilidad en $C$. hookerianum. No obstante, nada se sabe en relación a la otra especie presente en Chile. Por este motivo, el presente trabajo tiene por objetivo determinar el sistema reproductivo de C. patagua a fin de ampliar el conocimiento de la biología reproductiva de las especies del género presentes en Chile. Si las especies leñosas usualmente presentan sistemas reproductivos autoincompatibles (Barrett 1998) y C. hookerianum es autoincompatible, se espera entonces que $C$. patagua sea autoincompatible al igual que la especie congénere.

\section{MATERIALES Y METODOS}

ESPECIE Y SITIO DE ESTUDIO

Se trabajó con Crinodendron patagua Mol., un árbol endémico de Chile central, que habita principal- mente en quebradas húmedas (Rodríguez et al. 1983). Las flores, hermafroditas y solitarias, se componen de un cáliz irregularmente pentadentado, pubescente, y una corola con cinco pétalos libres subcarnosos de color blanco. El androceo está formado por 15 a 18 estambres, mientras que el ovario, súpero y pubescente, se encuentra rodeado por 8 a 10 glándulas nectaríferas. El fruto es una cápsula tri a tetra-locular leñosa, con dos a cinco semillas en cada lóculo. Florece en primavera desde octubre a diciembre y sus frutos maduran en otoño dispersando sus semillas en marzo (Rodríguez et al. 1983).

Se trabajó en la Reserva Nacional Roblería del Cobre de Loncha, desde noviembre de 2002 a marzo de 2003. La reserva se ubica en la VI Región, entre los $34^{\circ} 08^{\prime} \mathrm{S}$ y $71^{\circ} 01^{\prime} \mathrm{O}$, presentando una vegetación conformada principalmente por bosque esclerófilo y matorrales xéricos. El clima de la zona corresponde a templado cálido, característico de zonas mediterráneas (Hajek \& di Castri 1975). En la reserva, $C$. patagua se encuentra escasamente representado por sólo dos pequeñas poblaciones.

\section{SiSTEMA REPRODUCTIVO}

Para determinar el sistema reproductivo de Crinodendron patagua se realizaron cuatro pruebas consistentes en cruzamientos dirigidos, a través de los cuales se estimó el índice de autoincompatibilidad (Kearns \& Inouye 1993). Se contó también con un tratamiento de polinización natural para determinar la eficiencia de la reproducción efectuada naturalmente, además de tener un control de la eficiencia de los cruzamientos manuales. Cada tratamiento se realizó en cinco árboles adultos y en no menos de ocho flores por individuo. Para determinar si C. patagua es capaz de fructificar en ausencia de polen, se realizó la prueba de agamospermia, seleccionándose 48 flores al estado de yema, a las que se les extrajeron las anteras (emasculación) y embolsaron hasta la fase de fructificación para evitar la llegada de polinizadores.

Similarmente, para determinar si C. patagua es autógama, es decir si es capaz de formar frutos y semillas polinizándose con polen de la misma flor sin previa mediación de polinizadores, se realizó la prueba de autopolinización automática, embolsándose 43 yemas florales, sin emascular, las que se mantuvieron hasta la etapa de predispersión de 
semillas. Para evaluar geitonogamia, es decir, la capacidad de formar frutos y semillas con polen de la misma planta movilizado por los polinizadores, se realizó la prueba de autopolinización manual, para lo cual se embolsaron 47 yemas florales emasculadas. En el momento de la receptividad estigmática se procedió a cruzar manualmente las flores con polen proveniente del mismo individuo.

Para determinar xenogamia, es decir, la formación de frutos y semillas con polen proveniente de flores de otros individuos, se realizó la prueba de polinización cruzada manual. Para esto, se embolsaron 48 yemas florales emasculadas, las que al momento de la madurez sexual fueron cruzadas con polen exógeno proveniente de 4 individuos distintos distanciados por a lo menos $10 \mathrm{~m}$. Debido al escaso número de individuos en la población, el polen exógeno utilizado para los cruzamientos manuales provino de una distancia promedio de $6 \mathrm{~m}$. La eficiencia reproductiva natural se estimó a través de la prueba de polinización natural, para lo cual se marcó de forma inconspicua 42 yemas florales, permitiéndose el libre acceso de los polinizadores.
Los resultados de estos tratamientos se evaluaron mediante el recuento de frutos y semillas producidos al final de la temporada de fructificación. Estos frutos se mantuvieron embolsados hasta la etapa predispersiva para evitar pérdida por depredación o caída de los mismos por condiciones ambientales. Finalmente, se calculó el índice de autoincompatibilidad (IAI), que va de 0 (i.e., autoincompatibilidad) a 1 (autocompatibilidad), a través de la razón entre el número de semillas producidas por flor cruzada en la prueba de autopolinización manual y el número de semillas por flor cruzada producidas en la prueba de polinización cruzada manual (Ruiz-Zapata \& Arroyo 1978). La fructificación de las flores cruzadas experimentalmente y polinizadas naturalmente se comparó mediante una prueba de proporciones. Por su parte, el número de semillas producidas por fruto formado en las flores cruzadas experimentalmente se comparó con el número de semillas por fruto formado bajo polinización natural mediante la prueba no paramétrica de Mann-Whitney.

TABla I. Eficiencia reproductiva de Crinodendron patagua (promedio \pm ee).

Fructificación

\begin{tabular}{lccccc}
\hline Tratamiento & $\begin{array}{c}\mathrm{N}^{\circ} \\
\text { flores }\end{array}$ & $\mathrm{N}$ & $\%$ & $\begin{array}{c}\mathrm{N}^{\circ} \text { semillas } \\
\text { fruto }\end{array}$ & $\begin{array}{c}\mathrm{N}^{\circ} \text { semillas / flor } \\
\text { cruzada }\end{array}$ \\
\hline Agamospermia & 48 & 0 & 0,0 & $0,0 \pm 0,0$ & $0,0 \pm 0,0$ \\
$\begin{array}{l}\text { Auto- } \\
\text { polinización } \\
\text { automática }\end{array}$ & 43 & 3 & 6,9 & $7,3 \pm 2,3$ & $0,5 \pm 0,3$ \\
$\begin{array}{l}\text { Auto- } \\
\text { polinización } \\
\text { manual }\end{array}$ & 47 & 13 & 27,7 & $3,2 \pm 0,6$ & $0,9 \pm 0,3$ \\
$\begin{array}{l}\text { Polinización } \\
\text { cruzada } \\
\text { manual }\end{array}$ & 48 & 35 & 72,9 & $9,6 \pm 0,6$ & $7,0 \pm 0,8$ \\
$\begin{array}{l}\text { Polinización } \\
\text { natural }\end{array}$ & 42 & 31 & 73,8 & $7,6 \pm 2,3$ & $5,4 \pm 0,7$ \\
\hline
\end{tabular}




\section{RESULTADOS}

Las flores de la prueba de agamospermia no fructificaron, demostrando que la especie no es apomíctica al no ser capaz de fructificar y dar semillas en ausencia de polen (Tabla I). La fructificación por autopolinización automática fue 10,6 veces menor que la fructificación por polinización cruzada manual (Prueba de proporciones, $\mathrm{Z}=6,97 ; \mathrm{P}<0,005$ ), mientras que la fructificación por autopolinización manual fue 2,6 veces menor que la fructificación por polinización cruzada (Prueba de proporciones, $Z=$ $4,42 ; \mathrm{P}<0,005)$. Por otro lado, no hubo diferencias significativas entre el número de flores que fructificaron por polinización cruzada manual y polinización natural (Prueba de proporciones, $\mathrm{Z}$ = 0,$10 ; \mathrm{P}=0,460$ ).

El número de semillas por fruto (Tabla I) no difirió significativamente entre las pruebas de autopolinización automática y polinización cruzada manual (Prueba de Mann-Whitney, $\mathrm{U}=32,5 ; \mathrm{P}=$ $0,275)$. En cambio, el número de semillas producidas por fruto en la prueba de autopolinización manual fue tres veces menor que en la prueba de polinización cruzada (Prueba de Mann-Whitney, $\mathrm{U}=$ $36,5$; $\mathrm{P}<0,005)$. Por su parte, los frutos producidos por polinización natural tuvieron en promedio un $21 \%$ menos semillas que los frutos producidos por polinización cruzada manual (Tabla I: Prueba de Mann-Whitney, $\mathrm{U}=321 ; \mathrm{P}=0,003$ ).

La razón entre el número de semillas producidas por flor cruzada en la prueba de autopolinización manual y el número de semillas producidas por flor cruzada en la prueba de polinización cruzada manual (IAI) fue de 0,13 .

\section{DISCUSION}

De acuerdo al valor del índice de autoincompatibilidad obtenido para C. patagua, este árbol debe ser considerado autoincompatible sensu Richards (1986). Esta restricción que presentan los individuos para autofecundarse se expresa en una baja fructificación obtenida en la prueba de autopolinización manual, que se confirma con la fructificación aún menor obtenida en la prueba de autopolinización automática. La autoincompatibilidad observada en C. patagua va de acuerdo con la tendencia general observada que relaciona fuertemente el hábito de las plantas con el sistema reproductivo (Barrett 1998). De hecho, un alto número de plantas en los bosques templados de Chile requieren de la intervención de un agente polinizador biótico para su reproducción sexual, debido principalmente a su hábito leñoso (Arroyo \& Squeo 1990, Riveros et al. 1996).

Por otro lado, el menor número de semillas obtenidas por polinización natural respecto de la polinización cruzada manual sugiere que la producción de semillas en $C$. patagua puede estar limitada por la actividad de los polinizadores (Bierzychudek 1981). El movimiento de forrajeo de los polinizadores, quienes visitan numerosas flores por cada árbol individual, puede aumentar la incidencia de los cruzamientos geitonogámicos, con sus consecuentes efectos deletéreos para la producción de semillas. No obstante, es preciso ser cautos en esta conjetura, puesto que el depósito de polen ad libitum en los estigmas de las flores utilizadas para la polinización cruzada manual, puede haber estimulado una reasignación de recursos en la planta y por consiguiente, el menor número de semillas observado en la polinización natural, puede no ser el resultado de la actividad de los polinizadores (Zimmerman 1988).

El conjunto de características morfológicas de la flor y la producción de néctar de éstas pueden ser asociadas al síndrome de polinización por insectos (Proctor et al. 1996). Si bien no se efectuaron recuentos efectivos de la tasa de visitas de los polinizadores, se observó a Bombus dahlbomii y dípteros no identificados libando frecuentemente las flores de C. patagua. Estos insectos probablemente actúan como vectores polínicos entre los distintos individuos de la población. Por consiguiente, C. patagua debe considerarse como xenógama dada la dependencia que presenta de animales para la movilización de polen entre árboles distintos, y la consecuente polinización y producción de semillas.

\section{AGRADECIMIENTOS}

Agradecemos al personal de CONAF-VI Región por brindarnos su apoyo y amistad en nuestro trabajo en terreno, especialmente a los Sres. Allan Rojas, Roberto Cerda, Abel Orellana y Ricardo Muñoz. 


\section{REFERENCIAS}

ARRoYo, M.T.K. \& SQUEO, F.A. 1990. Relatioship between plant breeding systems and pollination. En: Kawano, S. (ed.). Biological Approaches and Evolutionary Trends in Plants. Pp. 205-227. Academic Press, London.

BARRETT, S.C.H. 1988. The evolution, maintenance, and loss of self-incompatibility systems. En: Lovett Doust, J. \& Lovett Doust, L. (Eds.). Plant Reproductive Ecology. Patterns and Strategies. Pp. 98-124. Oxford University Press, New York.

BARRETT, S.C.H. 1998. The evolution of mating strategies in flowering plants. Trends in Plant Science 3: 335341.

Bierzychudek, P. 1981. Pollinator limitation of plant reproductive effort. American Naturalist 117: 838840

BRICKER, J.S. 1991. A revision of the genus Crinodendron (Elaeocarpaceae). Systematic Botany 16: 77-88.

Charlesworth, D. \& Charlesworth, B. 1987. Inbreeding depression and its evolutionary consequences. Annual Reviews of Ecology and Systematics 18: 237-268.

Eckert, C.G. \& Barrett, S.C.H. 1994. Inbreeding depression in partially self-fertilizing Decodon verticillatus (Lythraceae): populations genetics and experimental analysis. Evolution 48: 952-964.

Frankel, R. \& Galun, E. 1977. Pollination Mechanisms,
Reproduction and Plant Breeding. SpringerVerlag, Berlin.

Hajek, E. \& Di Castri, F. 1975. Bioclimatología de Chile. Dirección de Investigación, Universidad Católica de Chile, Santiago, Chile.

Kearns, C.A, \& Inouye, D.W. 1993. Techniques for Pollination Biologists. University Press of Colorado. Colorado.

Morgan, M.T., Schoen, D.J. \& Bataillon, T.M. 1997. Evolution of self-fertilization in perennials. American Naturalist 150: 618-638.

Proctor, M., Yeo, P. \& Lack, A. 1996. The natural history of pollination. Timber Press. Oregon, Portland.

Richards, A.J. 1986. Plant Breeding Systems. Chapman \& Hall. London.

Riveros, M., Humaña, A.M. \& M.T. Arroyo. 1996. Sistemas de reproducción en especies del bosque valdiviano ( $40^{\circ}$ latitud sur). Phyton 58: 167-176.

Rodríguez R., Matthei, O. \& QuezadA, M. 1983. Flora Arbórea de Chile. Editorial de la Universidad de Concepción, Concepción.

Ruiz-Zapata, T. \& Arroyo, M.T.K. 1978. Plant reproductive ecology od a secondary deciduous forest in Venezuela. Biotropica 10: 221-230.

Zimmerman, M. 1988. Nectar production, flowering phenology, and strategies for pollination. En: Lovett Doust, J. \& Lovett Doust, L. (eds.). Plant Reproductive Ecology. Patterns and Strategies. Pp. 157-178. Oxford University Press, New York.

Fecha de recepción: 25.05 .04

Fecha de aceptación: 24.08.04 